
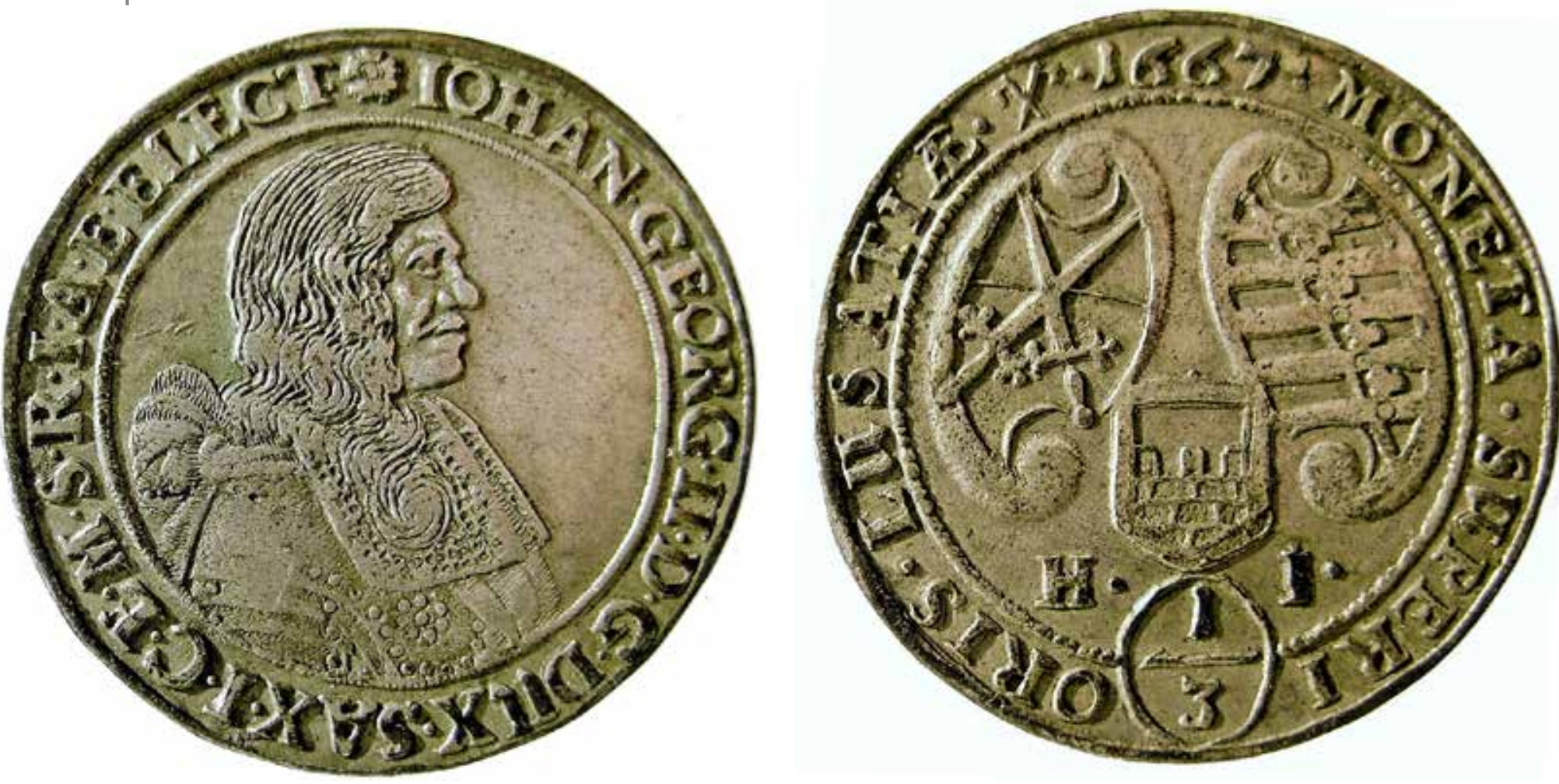

\title{
Numismatische Streiflichter zur Stadt Löbau
}

\author{
Ewald Hausmann und Lars-Gunther Schier
}

Dritteltaler Kurfürst Johann Georg II. (1656-1680)

Vorderseite: Brustbild mit

Allongeperücke nach rechts.

Umschrift: C IOHAN GEORG II.•G

$\bullet D V X \bullet S A X \bullet \mid \bullet C \bullet E \bullet M \bullet S \bullet R \bullet 1 \bullet A \bullet$

E•ELECT

Rückseite: Das Wappen

der Oberlausitz zwischen

Kur- und Rautenwappen,

darunter das Kürzel $\mathrm{H} \bullet \mathbf{I} \bullet$ des

Münzmeisternamens.

Umschrift: • $1667 \bullet$ MONETA •

SUPERI ( $1 / 3$ ) ORIS • LUSATI/E

Die gekreuzten Zainhaken im

Abschluss sind das Zeichen des

Münzmeisters.
Speziell für Löbau geschaffenes Geld gab es nur in einer Ausnahmesituation für sehr kurze Zeit. Übliche Währung waren die Ausgaben des jeweiligen Landesherren für die ganze Oberlausitz und später darüber hinaus für Böhmen, für Sachsen, für Deutschland und für die Eurozone. Die Verleihung des Stadtrechts an Bautzen (1213), Kamenz (1225), Löbau (1238), Zittau (1255) und Görlitz (1303) förderte deren Entwicklung als Marktorte. Der beginnende Warenverkehr erforderte Zahlungsmittel. Die königlich böhmischen Städte erhielten das auf ihren Märkten gültige Geld aus der Münzstätte Bautzen, die schon für den Markgrafen von Meißen und den Kaiser Friedrich I. tätig gewesen war. Später kamen zeitweilig auch Pfennige aus Görlitz und Zittau hinzu.

Der 1300 eingeführte Prager Groschen verbreitete sich aus Böhmen auch in den Nachbarländern und war für die Oberlausitz die erste überregionale Münze. Die nächsten waren der Meißner Groschen und der Taler aus St. Joach- imsthal und Annaberg. Kursachsen hatte 1635 beide Lausitzen erworben.

Die Oberlausitz hatte zunächst noch einen Sonderstatus behalten. Daraus leitete Kurfürst Johann Georg II. von Sachsen 1666 ein Recht auf die Ausgabe einer sogenannten Landmünze ab. Diese Münze, deren Silbergehalt nicht der Vorschrift der Reichsmünzordnung genügte, war ebenso unterwertig wie die Ausgaben der Nachbarn Sachsens - und wie die des Böhmenkönigs, des früheren Landesherren. Das eingesparte Silber sollte einen zusätzlichen Münzgewinn erbringen.

Nominell war diese Münze, wie die Umschrift der Rückseite besagt, MONETA SUPERIORIS LUSATIÆ, Geld der Oberlausitz, obwohl es für den Umlauf in ganz Sachsen gedacht war. Für die Herstellung wurde eigens eine Münzstätte in Bautzen eingerichtet. Im sächsischen Stammland wurde dieses Geld aber abgelehnt. Man bestand auf Bezahlung in vollwertiger Münze. Darum und weil der erhoffte Gewinn sich in engen 


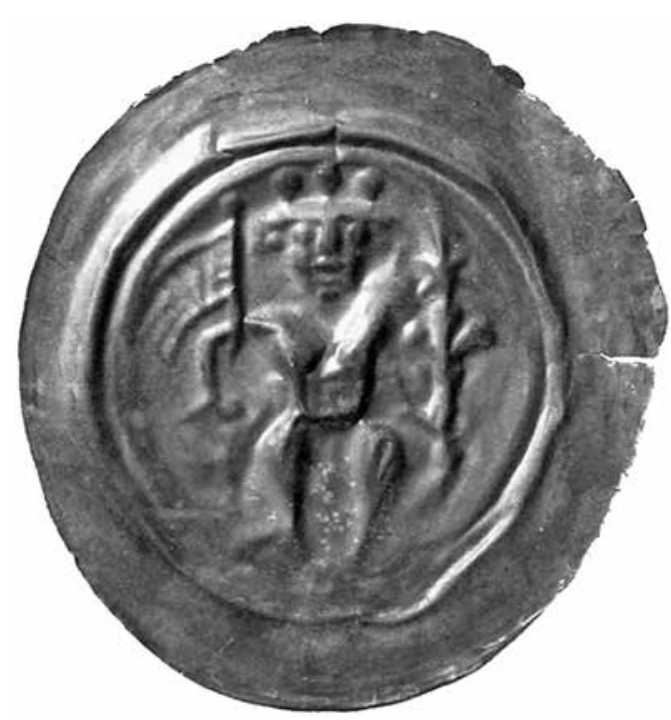

Grenzen hielt, brach der Kurfürst das Unternehmen mit dem zweiten Jahrgang ab.

Zur Ausgabe eigenen Löbauer Geldes kam es zum Ende des Ersten Weltkriegs und unmittelbar danach. Die bis 1914 den Zahlungsverkehr beherrschenden Gold- und Silbermünzen wurden für Importe aus dem neutralen Ausland benötigt. Sie wurden - anfangs mit Erfolg - im Zahlungsverkehr durch Banknoten ersetzt. Problematisch wurde der Kleingeldmangel, denn die Prägung von Kleinmünzen wurde zunehmend eingeschränkt. Das ursprünglich dafür eingesetzte Material (Kupfer und Nickel) wurde in die Rüstungsindustrie gelenkt, und die „Ersatzmetalle“ Eisen, Zink und Aluminium machten zunächst Schwierigkeiten bei der Verarbeitung. Beinahe zwangsläufig wurde das „richtige Geld“ gern gehortet, soweit man sich das leisten konnte, und fehlte im Umlauf.

Im Deutschen Reich war die Ausgabe von Banknoten und Münzen nur der Reichsbank und einigen Landesbanken erlaubt. Diese waren nicht mehr in der Lage, genügend Kleingeld zur Verfügung zu stellen. Notgedrungen übernahmen Kommunen, Banken und Händler die Bereitstellung von Ersatz-Zahlungsmitteln. Spätestens 1917 wurden Gutscheine, Wertscheine $u$. dgl. für Pfennigbeträge in Umlauf gebracht. Häufig gaben die Stadtverwaltungen - wie in Löbau - die ersten Notgeldscheine aus. Die Stadtkasse garantierte mit ihren Beständen die Einlösung, weshalb diese Scheine innerhalb der Stadt allgemein akzeptiert wurden. Gutscheine über 10 Pfennige rechtfertigten 1917 noch einigen Aufwand in der Herstellung. Sechs Jahre später waren sogar Reichsbanknoten über Milliardenbeträge grafisch einfach gestaltet und nur noch einseitig bedruckt.

Vom April 1917 bis Mai 1921 wurden sieben Auflagen des 10-Pfennig-Scheins ausgegeben.

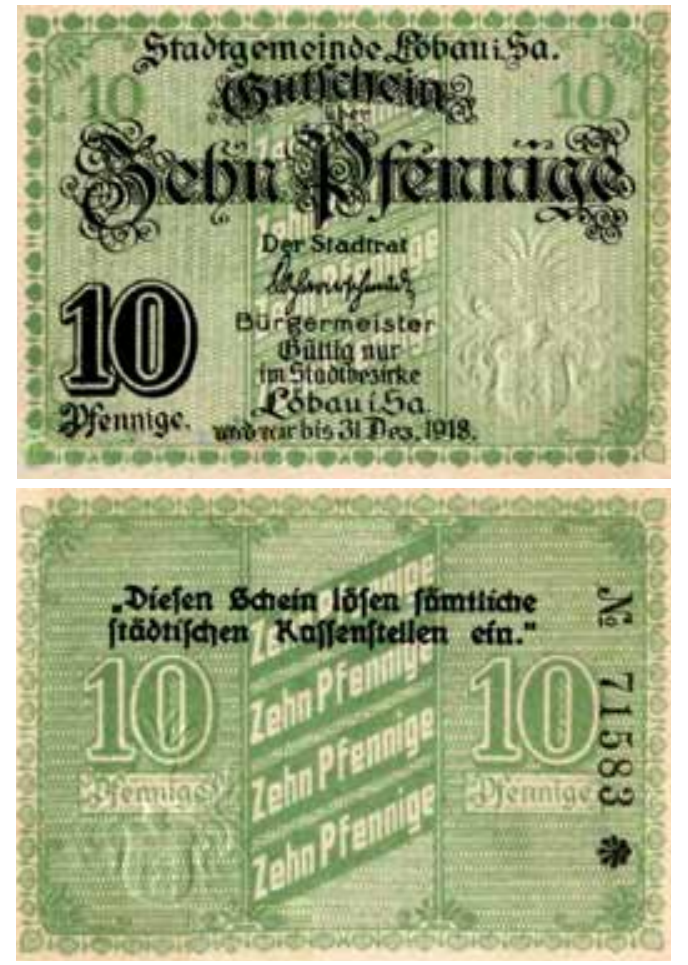

Das Druckbild blieb unverändert, obwohl die Geltungsdauer sich schon in der 4. Auflage änderte. Die Korrektur erfolgte wohl als Mitteilung in der Presse. Ein ähnlich gestalteter Schein in der Wertstufe 50 Pfennig erlebte ebenfalls 7 Auflagen.

Schon 1920 wurden kleine Pfennig-Beträge, wie die über 1 und 2 Pfennig, wesentlich einfacher gestaltet. Sie waren aber immerhin auf Karton gedruckt und damit für einen mehrfachen Kreislauf geeignet.

Der Consum-Verein Löbau gab ebenfalls Wertmarken aus, und zwar in den Wertstufen 1, 5, 10 und 30 Pfennig, die auch in Konsum-Geschäften der Umgebung angenommen wurden. Geprägte Wertmarken wirken wohl eher wie Geld als die die gedruckten Exemplare. Allerdings stand für die Bemühungen des ConsumVereins weder genügend geeignetes Material noch leistungsfähige Stanz- und Prägetechnik zur Verfügung. Aus dünnem Zink- bzw. Eisenblech wurden einseitige Marken in den Wertstufen 5, 10, 20, 50 und 100 Pfennig geschlagen, die die technischen Probleme der Herstellung deutlich erkennen lassen.

Im Deutschen Reich war die Ausgabe von Banknoten und Münzen nur der Reichsbank und einigen Landesbanken erlaubt. Als die Inflation an Tempo zunahm, gab die Reichsbank Banknoten mit zunehmend höheren Nominalen aus. Mit dem rapiden Wertverfall des Geldes konnten ihre Druckereien damit aber nicht Schritt halten. Die Kommunen waren wieder zur Selbsthilfe genötigt. Statt der überforderten
Pfennig in Form eines Brakteaten, König Wenzel I. von Böhmen (1223-1253)

Die einseitige Prägung stellt den sitzenden König dar, der sich mit der Fahne als Lehnsmann des deutschen Königs bezeichnet. In der Linken hält er ein Blumenzepter als Herrschaftssymbol.

10-Pfennig-Schein, 1918

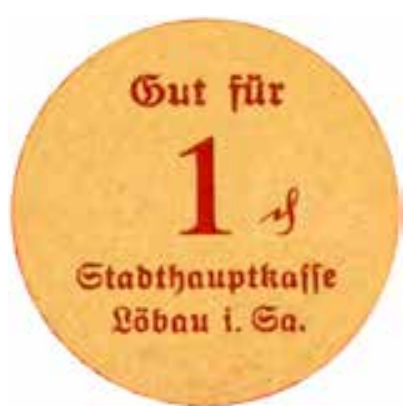

Dieser Pfennig, ausgegeben 1920, galt nur in der Stadt Löbau, wo er auch hergestellt wurde. Zweipfennigstücke sind auf grünem Karton gedruckt. Die Rückseite trägt den Stempel der Stadthauptkasse und eine Unterschrift.

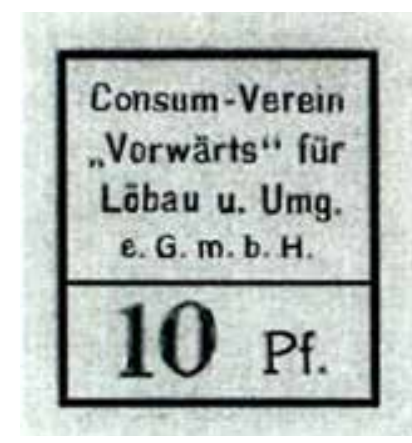

Die Konsumgenossenschaft sah sich zur Ausgabe eigener Zahlungsmittel genötigt 


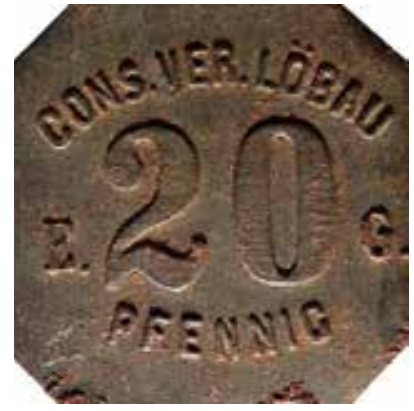

Hohlprägungen von dünnem Blech waren sogar in Eisen mit begrenztem Kraftaufwand möglich. Die Stempel brauchten nur den Wert und den Herausgeber zu nennen.
Stadtkasse übernahmen ortsansässige Kreditinstitute die Versorgung mit Geldscheinen. Vom 2. August 1923 bis zum 18. Oktober 1923 gab die Stadtgirokasse Scheine in den Wertstufen Einhunderttausend bis Fünfhundert Milliarden Mark aus. Die Gültigkeitsdauer ergab sich schon bald aus dem Kursverfall und dem Datum der Einlösung, das die Wertstellung bestimmte.

Die Stadtsparkasse und Banken wie die Commerzbank und die Allgemeine Deutsche Creditbank stellten ihren Kunden Schecks mit eingedruckten Beträgen als Zahlungsmittel zur Verfügung, die „Nur zur Verrechnung“ bestimmt waren. Der abgebildete Schein steht für folgenden Sachverhalt: Ein Kunde der Sparkasse hob von seinem Konto zehn Milliarden Mark ab. Den Betrag erhielt er in Form eines Schecks, mit dem er etwa einen Wocheneinkauf an Lebensmitteln für eine dreiköpfige Familie bezahlte. Der Händler trug den Scheck möglichst umgehend zur Stadtgirokasse, wo dieser seinem Konto zum Tageskurs gutgeschrieben wurde.

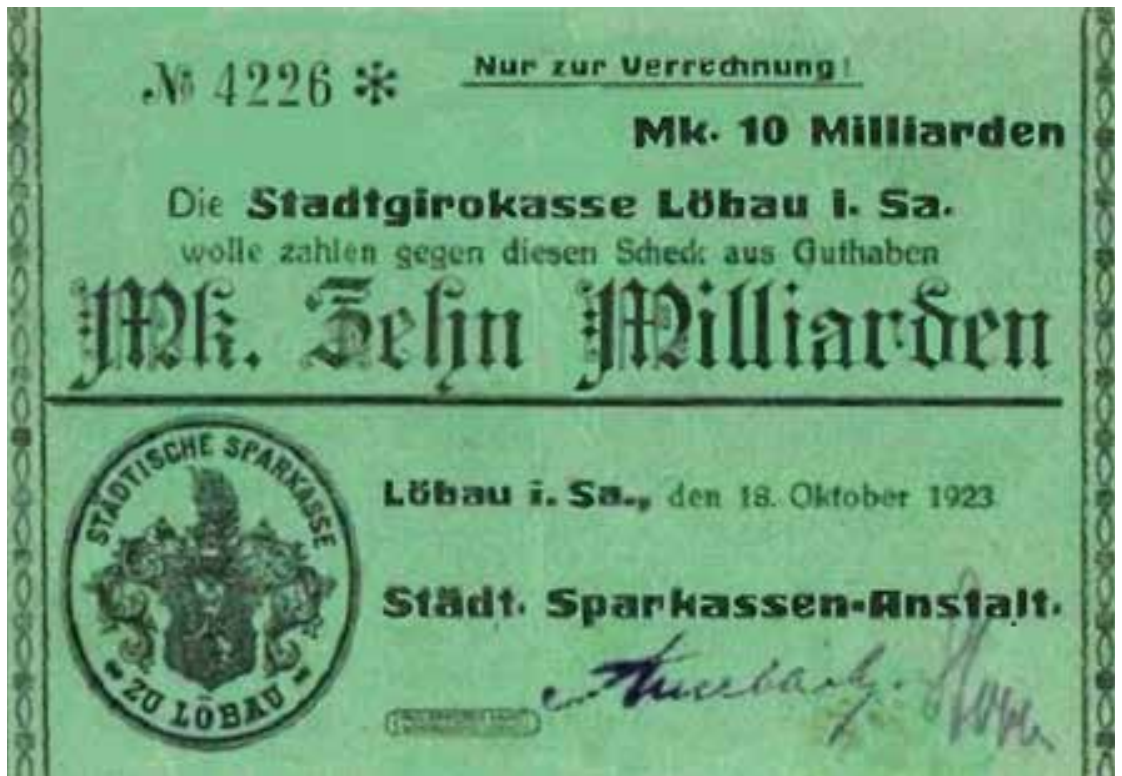

Verrechnungsscheck über zehn Milliarden Mark
Im Bereich der Amtshauptmannschaft verlief die Entwicklung analog. In der Zeit des schnellsten Verfalls der Währung verfiel die ConsumGenossenschaft auf eine Art wertbeständigen Notgelds in Form von Waren-Gutscheinen. Helfricht stellt Beispiele für Brot- und Buttermarken vor, die offenbar auf die Rückseite von Eintrittskarten gedruckt wurden.

\section{Löbauer Traditionspflege durch Ausgabe von Medaillen}

Die Geschichte der Stadt, Schulen, Vereine, besondere Gebäude, Feste u. a. m. lieferten Anlässe und Motive für eine Vielzahl von Medaillen- ausgaben. Das 700-jährige Jubiläum der Verleihung des Stadtrechts wurde mit einer Serie von Prägungen der Meißner Porzellanmanufaktur aus Böttgersteinzeug gefeiert. 50 Jahre später wurden ebenfalls drei Varianten aus diesem Material gefertigt.

Auf der Vorderseite umschließen zwei Zweige ein Quadrat mit der Inschrift LÖBAU / 700 / JAHRE / STADT. Die Rückseite präsentiert das Stadtwappen, darüber STADT LÖBAU. Darunter die Meißner Schwerter zwischen den Jahreszahlen 1221 und 1921.

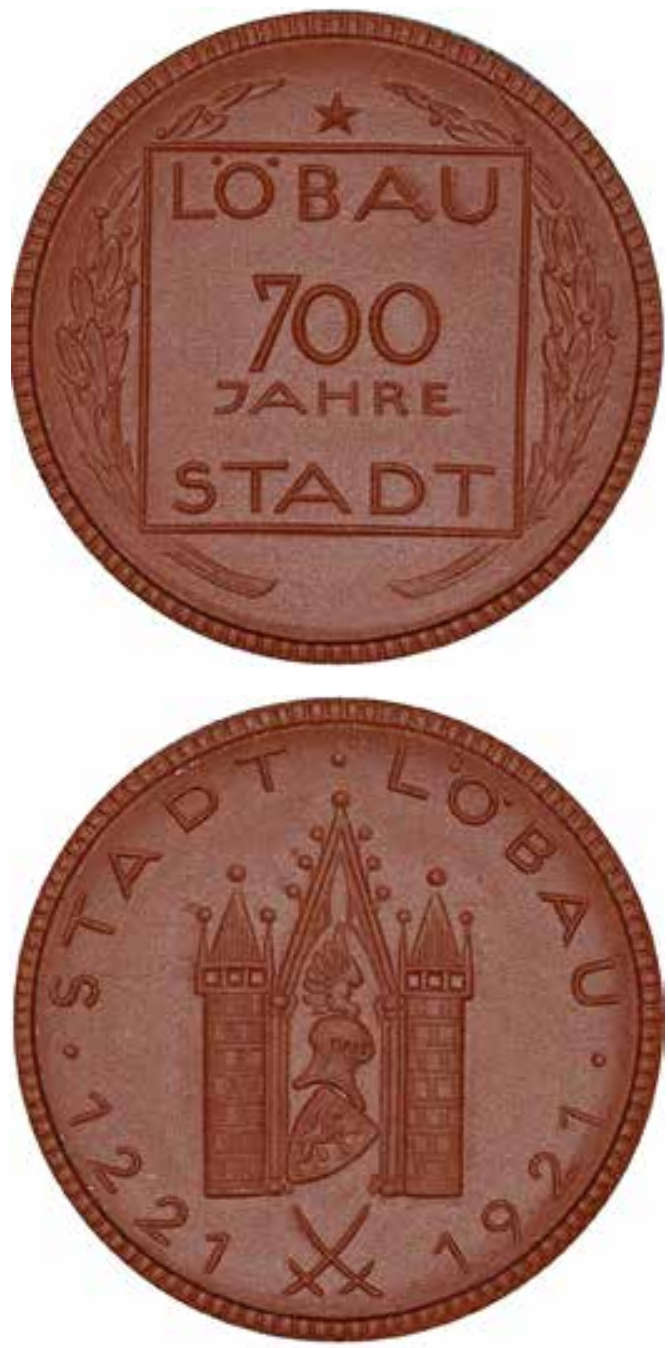

Mit einem anderen Motiv erschienen Ausgaben in drei verschiedenen Metallen.

Die 775-Jahr-Feier der Stadt wurde gemeinsam mit dem 650. Jahrestag des Gründungskonvents des Sechsstädtebunds begangen. Mit dem Pokal, der im Ritual der Konvente der Städtevertreter eine Rolle spielte, erinnert Löbau an die große Vergangenheit als Mitglied des Städtebundes.

Die Vorderseite zeigt zwischen der Zahl 650 und dem Wort JAHRE diesen Pokal aus Böhmischem Glas, der im 17. Jh. verloren gegangene Vorgänger ersetzte. Die (farbigen) Ornamente umrahmen 
die Wappen der sechs Städte. Die Umschrift nennt den OBERLAUSITZER SECHSSTÄDTEBUND.

Auf der Rückseite der Medaille stehen die Wappen der Bundesmitglieder. Da die Gründung wie die meisten Routinesitzungen in Löbau stattfanden, wird dieses Prunkstück im Museum der Stadt bewahrt.

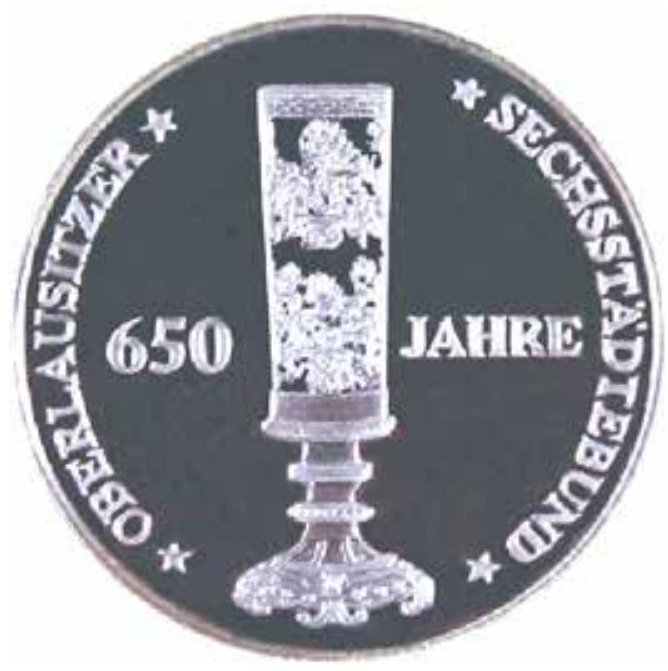

Der 1854 eingeweihte eiserne Aussichtsturm wurde rasch zum Wahrzeichen der Stadt. Der König hatte ihn als persönliches Denkmal akzeptiert. Gleichzeitig ist der Turm auch ein Denkmal für die Großzügigkeit des Bäckermeisters, der die Finanzierung des Baus sicherte.

Auf der Rückseite steht das gekrönte Monogramm des Königs in einem Kranz, umgeben von

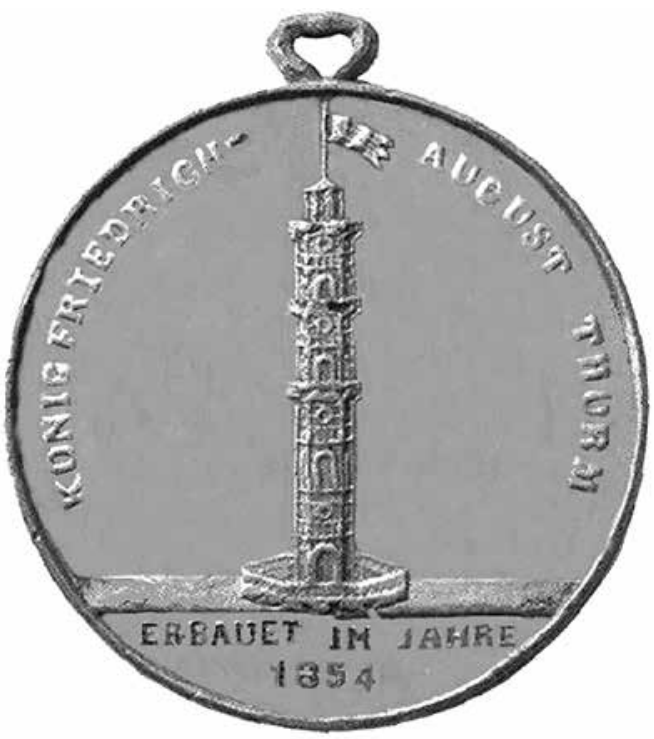

der Widmung in zwei Zeilen: HIER VON DEN EHERNEN ZINNEN ERBLICKST DU DIE HERRLICHKEIT GOTTES UND IN DER STERBLICHEN KUNST ZEIGT SICH D. GÖTTLICHE GEIST. In späteren Jahren war der Turm mehrmals Motiv einer Medaillenprägung.
Das Königliche Lehrerseminar war die erste moderne Höhere Lehranstalt in der Stadt. Nicht die Einweihung der 1873 gegründeten Schule, sondern die Weihe der Schulfahne war 1887 Anlass für zwei Medaillenausgaben. Heute beherbergt der Gebäudekomplex das Gymnasium. Die Medaillen wurden aus Messing hergestellt. Die Rückseite zeigt das Königlich Sächsische Wappen.

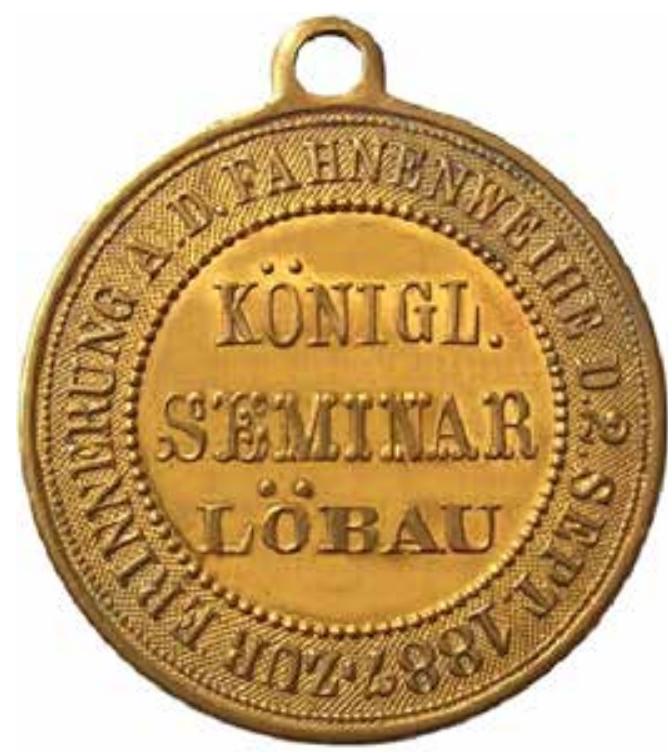

Die 1963 gegründete Offiziersschule des Heeres wurde 1971 zur Hochschule. Damit war in Löbau die höchste Bildungsstufe erreichbar. Sie feierte ihr 15-jähriges Bestehen mit dieser Ausgabe. Sportliche und militärische Wettkämpfe waren Anlässe weiterer Emissionen. Auf der Rückseite der Medaille erscheint der Kopf des Namengebers der Hochschule, Ernst Thälmann.

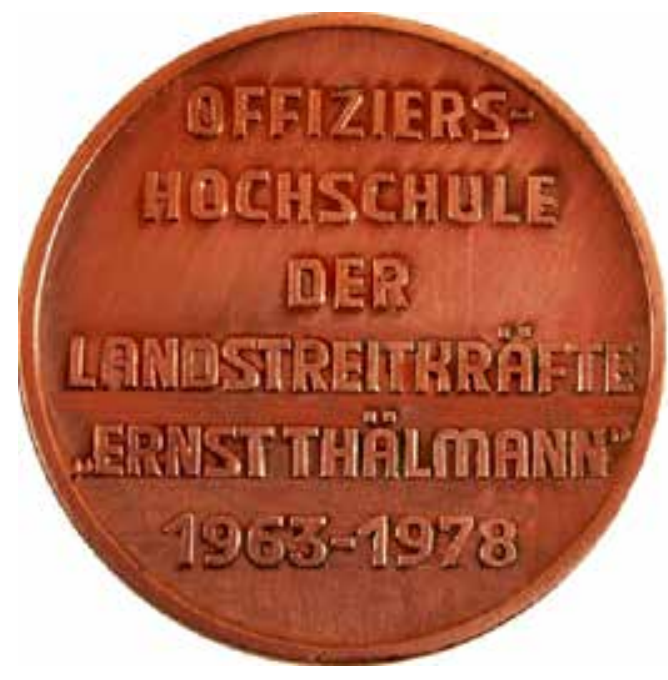

Der Wettin-Schützenbund war ein sachsenweiter Zusammenschluss von Schützenvereinen. Alle zwei Jahre hielt er in wechselnden Städten Wettkämpfe ab. 
Quellen

Peter Helfricht: Notgeldkatalog - Löbau/Sa. 1917-1923, Löbau 2012 mit Ergänzung 2014

Peter Helfricht: Löbau auf Medaillen, Abzeichen und anderen Gedenkartikeln

Walter Haupt: Sächsische Münzkunde, Textband S. 146148, Tafelband Tf. 100-102, Berlin 1971.

Klaus Thieme: Brakteaten der Markgrafschaft Meißen und ihrer Nachbarn zwischen Saale und Neiße, Leipzig 2011, S. 224 ff

Walter Haupt: Abriß der Münzprägung und des Geldumlaufes der Oberlausitz, in: Lars-Gunter Schier (Redaktionelle Leitung): Studien zur Oberlausitzer Numismatik, Krobnitz 2015, S. 1532, hier S. 19.

Lars-Gunter Schier: Aussichtstürme im Medaillenbild, in: Lars-Gunter Schier (Hrsg.): Studien zur Oberlausitzer Numismatik, Krobnitz 2015, S. 325334, hier S. 327.

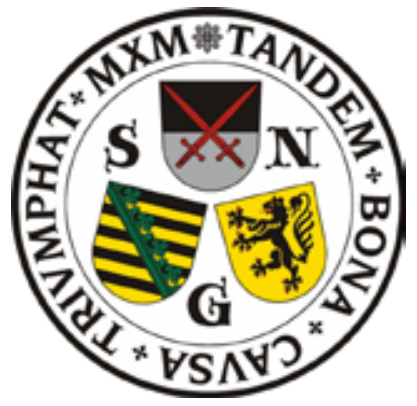

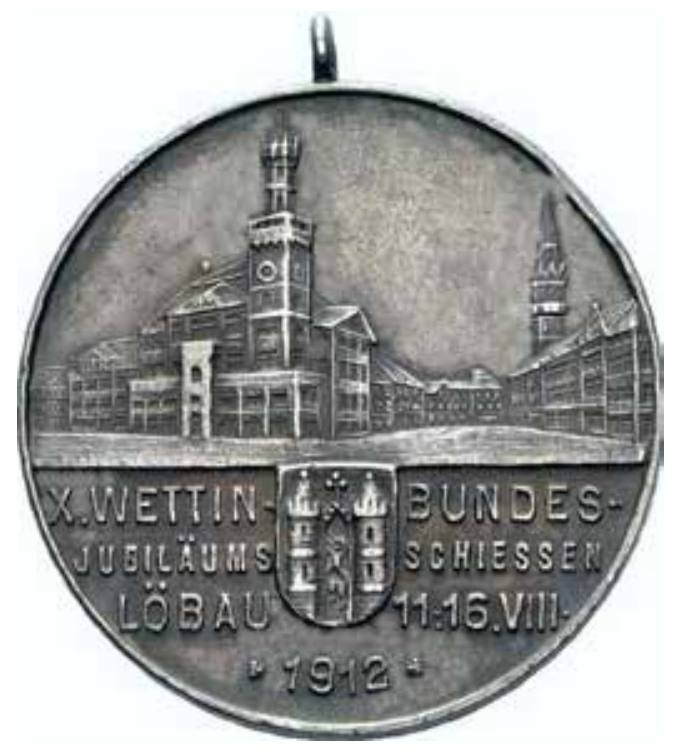
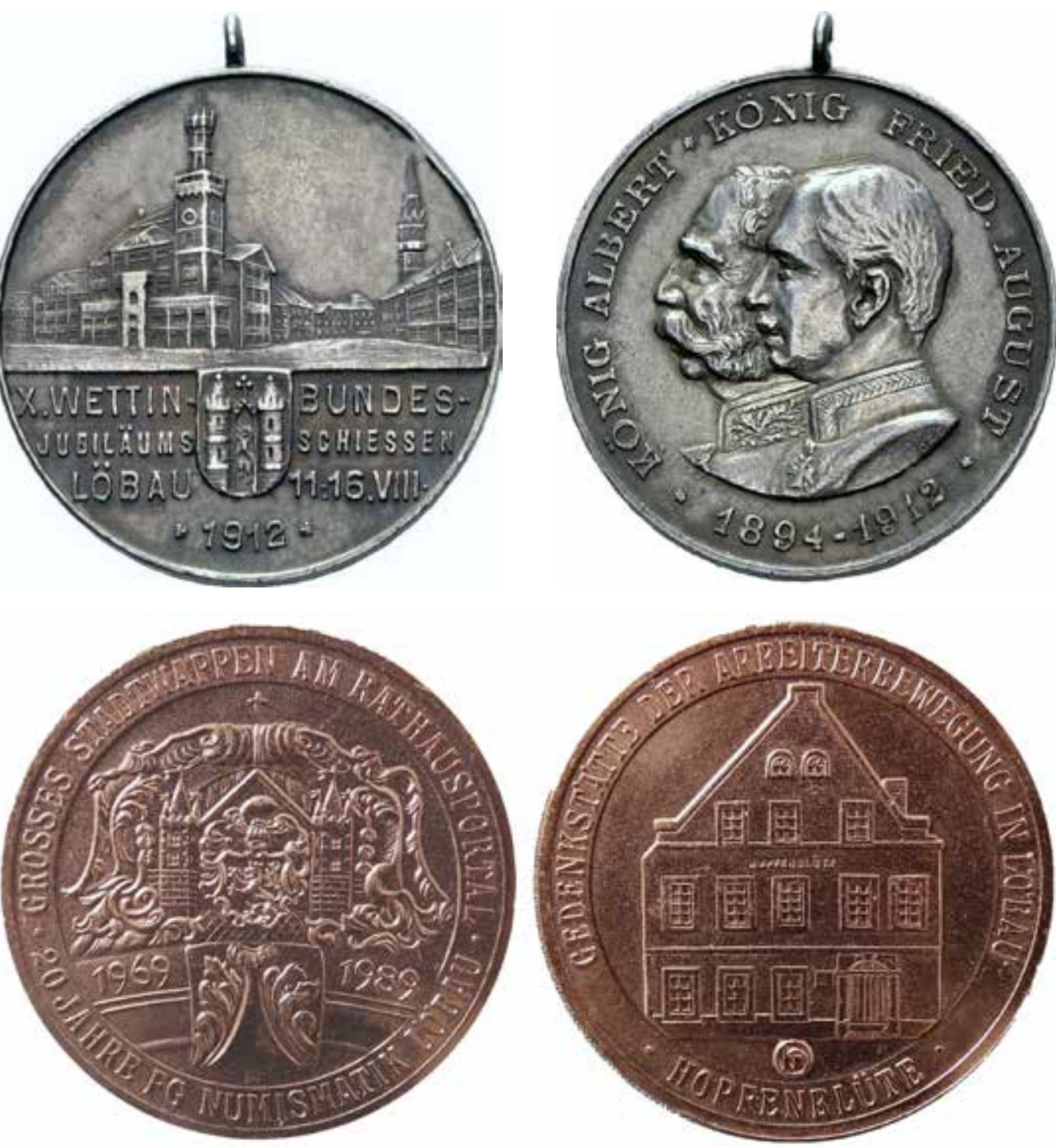

Auf der Vorderseite erscheinen Gebäude der Gastgeberstadt, dabei das Rathaus und die Nikolaikirche. Im Abschnitt teilt das Stadtwappen den Text X. WETTIN - BUNDES / JUBILÄUMS -SCHIESSEN / LÖBAU 11. - 16. VIII. / 1912. Die Rückseite zeigt die Könige Albert und Friedrich August III. im Brustbild.

Zum 20-jährigen Bestehen der Fachgruppe Numismatik des Kulturbundes der DDR wurde 1989 eine Medaille herausgegeben. Die Vorderseite zeigt das Große Wappen der Stadt Löbau zwischen den Daten 1969 und 1989, darunter das Signum MV. Umschrift: GROSSES STADTWAPPEN AM RATHAUSPORTAL • / 20 JAHRE FG NUMISMATIK LÖBAU. Auf der Rückseite ist die Fassade der ehemaligen Gaststätte „Hopfenblüte“, seit 1988 Gedenkstätte der Arbeiterbewegung, abgebildet. Umschrift: GEDENKSTÄTTE DER ARBEITERBEWEGUNG IN LÖBAU • / HOPFENBLÜTE • Der Entwurf stammt von Michael Vogt; Hersteller war der VEB MedaillenMünze Dresden. Es existieren 3 Varianten aus vermessingtem, vernickeltem und verkupfertem Eisen.

Festmedaille der Sächsischen Numismatischen Gesellschaft zum Tag der Sachsen

$\mathrm{Zu}$ den sächsischen Vereinen und Verbänden, welche alljährlich am ersten Septemberwochenende den Tag der Sachsen veranstalten, gehört von Anbeginn 1992 in Freiberg die Sächsische Numismatische Gesellschaft e.V., der Dachverband aller Münzfreunde und Münzsammler in Sachsen. Von Anbeginn gibt die Gesellschaft im Bunde mit den Veranstaltern auch eine offizielle Festmedaille heraus. Ein schöner Brauch, welcher Deutschlands größte fortlaufende Medaillenserie zu einem gesellschaftlichen Höhepunkt eines Bundeslandes entstehen ließ. Sie erfreut sich jedes Jahr bei Sammlern und Besuchern großer Beliebtheit.

Für Löbau legten die sächsischen Münzfreunde nun zum 26. Male eine solche Medaille auf. In 

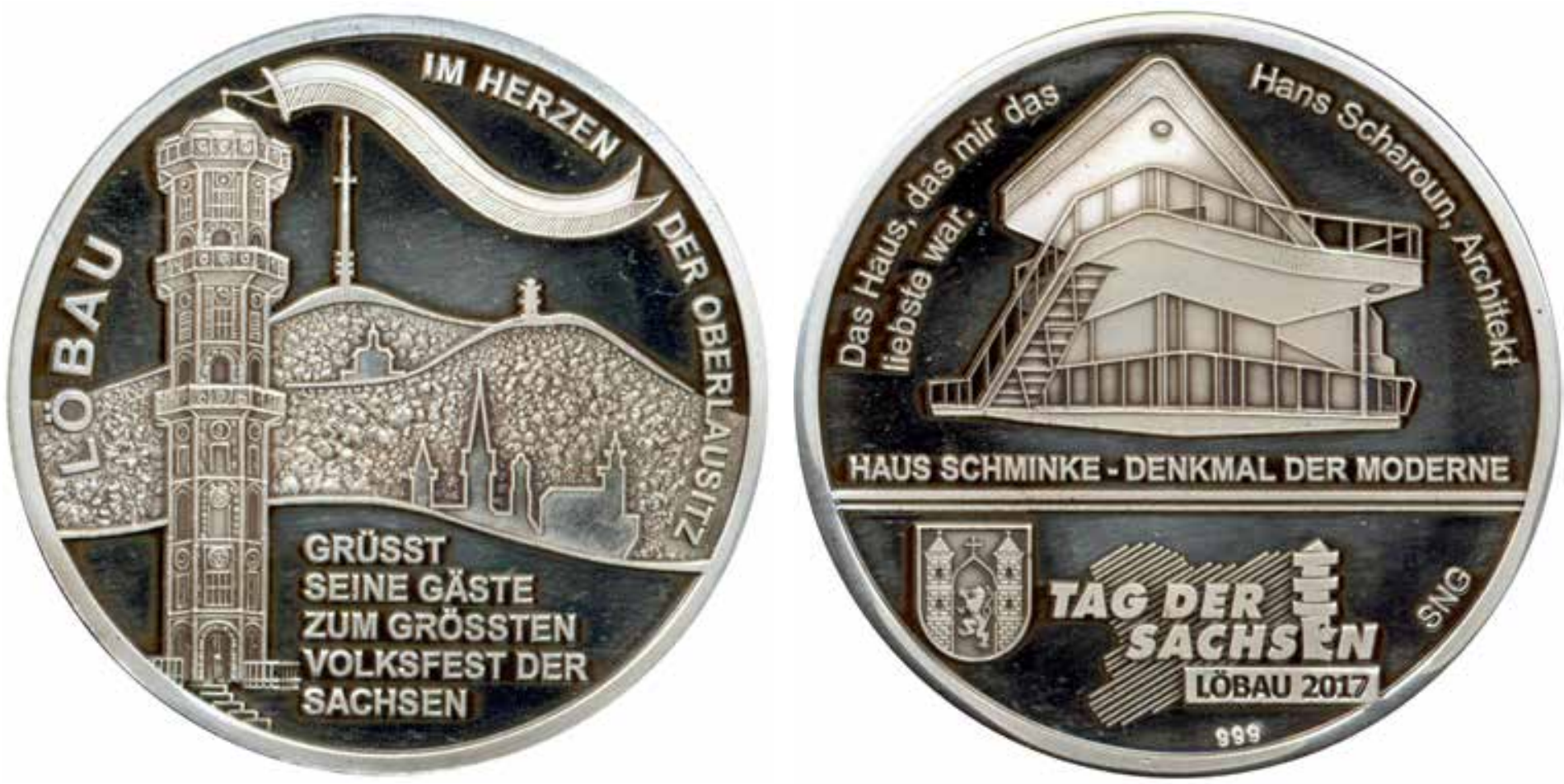

edlem Silber und anderen Materialien geprägt, verleiht sie dem 26. Tag der Sachsen wiederum ein würdiges und dauerhaftes Andenken. Die verwendeten Bildmotive spiegeln in bewährter Weise Highlights und Besonderheiten des Veranstaltungsortes und zusammen mit dem Motto „Löbau im Herzen der Oberlausitz“ ist sie ein schöner Botschafter für die freundliche Gastgeberstadt Löbau.

Die Festmedaille zeigt im Avers die Silhouette der Stadt Löbau, wie sie sich mit Kirchtürmen und Rathaus malerisch an den Löbauer Berg schmiegt und ihrem Beinamen „Stadt am Berge" gerecht wird. Auf der linken Bergkuppe ragt der $162 \mathrm{~m}$ hohe Sendeturm empor, auf der anderen Seite steht der historische Löbauer Turm, dazwischen das Bergrestaurant Honigbrunnen. Der filigrane gusseiserne Löbauer Turm - das Wahrzeichen der Stadt - zeigt sich dem Betrachter nochmals in voller Größe am linken Bildrand. Die Aussicht vom Löbauer Berg, einem einstigen Vulkan, ist ob seiner exponierten Lage grandios. Der Turm wurde bereits 1854 als Friedrich-August-Turm im Gedenken an den sächsischen König errichtet, er gilt heute als weltweit einziger gusseiserner Aussichtsturm und als schönster Turm Sachsens. Stolz weht auf ihm am Tag der Sachsen zum Gruße die weiß-grüne Fahne. Der Slogan „Löbau im Herzen der Oberlausitz“ ist schon über 600 Jahren alt, als der Oberlausitzer Sechsstädtebund (Kamenz, Bautzen, Löbau, Görlitz, Lauban und Zittau) die Stadt dank seiner zentralen Lage zum
Konventort erhob. Im September 2017 ist die Stadt Löbau nun abermals ein freundlicher Konventort - diesmal für ihre Gäste aus ganz Sachsen und darüber hinaus.

Der Revers der Medaille zeigt ein architektonisches Juwel der Stadt Löbau - das Haus Schminke. Beeindruckt von seinem Baustil der Moderne ließ sich Nudelfabrikant Fritz Schminke 1930 vom Berliner Architekten Hans Scharoun (1893-1972) ein extravagantes, aber funktionelles Wohnhaus entwerfen. Mit geschwungenen Linien und offenen Raumstrukturen erschuf Scharoun eine einzigartige lichtdurchflutete Symbiose mit der Natur. Das Haus, welches selbst dem Architekten Zeit seines Lebens das liebste war, gehört $\mathrm{zu}$ den bedeutendsten Architekturschöpfungen Deutschlands zwischen den Weltkriegen. Es ist heute Museum, Herberge und Eventstätte zugleich. Der untere Teil der Medaille trägt die Symbole der Herausgeber - das Wappen der Stadt Löbau, das Signet des Kuratoriums Tag der Sachsen und SNG als Kürzel der Sächsischen Numismatischen Gesellschaft.

Die 40 Millimeter große Medaille wurde in Feinsilber (patiniert), Bronze (vergoldet) und Kaiserzinn in der höchsten Prägequalität "Polierte Platte" von der 1. Dresdner Medaillenmünze Glaser \& Sohn GmbH hergestellt. Der Entwurf stammt von Lars-Gunter Schier (Seifhennersdorf), die Gravur erledigte Henning Firlle (Dresden). Die Auflage ist auf jeweils 300 Stück je Metallvariante limitiert.
Auf der Vorderseite die Silhouette der "Stadt am Berge", vor ihr ein detailreiches Bild des Eisenturmes und ein Begrüßungstext. Auf der Rückseite das Haus Schminke, ein Denkmal für modernes Bauen, neben dem Stadtwappen das Logo des Kuratoriums Tag der Sachsen und das Kürzel SNG (Sächsische Numismatische Gesellschaft) des Mitherausgebers der Serie.

\section{Autoren}

Ewald Hausmann

Lichtenau

Lars-Gunter Schier Seifhennersdorf 